\title{
ANSIEDAD Y VIDA COTIDIANA COMO BLANCOS FARMACOLÓGICOS EN URUGUAY 2013-2015
}

ANSIEDADE E VIDA COTIDIANA COMO ALVOS FARMACOLÓGICOS NO

URUGUAI 2013-2015

\author{
ANXIETY AND EVERTDAY LIFE AS PHARMACOLOGICAL TARGETS \\ IN URUGUAY 2013-2015
}

\author{
Andrea Biellii ${ }^{1}$, Pilar Bacci ${ }^{1}$, Gabriela Bruno ${ }^{1}$, Nancy Calisto ${ }^{1}$ e \\ Santiago Navarro ${ }^{1}$
}

${ }^{1}$ Universidad de la República, Montevideo, Uruguay

\begin{abstract}
RESUMEN: El artículo describe y analiza algunos procesos por los que la ansiedad y la vida cotidiana son transformadas en blancos farmacológicos por los profesionales de salud pública en Uruguay. Durante 2013 y 2014 se realizaron entrevistas en profundidad a 10 informantes calificados, 35 profesionales de salud pública (médicos generales, de familia, psiquiatras, psicólogos), dos grupos de discusión (uno con médicos generales y de familia; otro con psicólogas) y un relevamiento de literatura nacional sobre el tema. Se identificó el cruce de dos concepciones de ansiedad: una perteneciente al campo médico, sustentada en conceptos distantes de la experiencia, y otra perteneciente al campo social, sustentada en la experiencia de los profesionales con sus pacientes. Se concluye que su enlace con las denominadas "hipótesis sociológicas" de la ansiedad posibilita transformar problemas cotidianos y derivados de la situación socioeconómica apremiante que viven los pacientes, en problemas pasibles de tramitación farmacológica mediante benzodiazepinas.
\end{abstract}

PALABRAS CLAVE: Ansiedad; Benzodiazepinas; Psicofarmacología; Vida cotidiana.

RESUMO: Este trabalho descreve e analisa os processos pelos quais ansiedade e vida cotidiana são transformadas em alvos farmacológicos pelos profissionais de saúde pública do Uruguai. Durante 2013 e 2014 foram realizadas entrevistas em profundidade a 10 informantes qualificados, 35 profissionais de saúde pública (médicos gerais, de família, psiquiatras, psicólogos), dois grupos de discussão (um com médicos gerais e de família; outro com psicólogas) e revisão de bibliografia nacional sobre o tema. Identificou-se um cruzamento de duas concepções de ansiedade: uma pertencente ao campo médico, sustentada por conceitos distantes da experiência, e outra pertencente ao campo social, sustentada pela experiência de profissionais com seus pacientes. Conclui-se que sua vinculação com as chamadas "hipóteses sociológicas" da angústia possibilita a transformação de problemas cotidianos e derivados da situação socioeconômica imperiosa vivida pelos pacientes em problemas passíveis de processamento farmacológico mediante benzodiazepinas. PALAVRAS-CHAVE: Ansiedade; Benzodiazepinas; Psicofarmacologia; Vida cotidiana.

ABSTRACT: This paper describes and analyzes some processes by which anxiety and daily life are transformed into pharmacological targets by public health professionals in Uruguay. Through 2013 and 2014 we carried out indepth interviews with 10 qualified informants, 35 public health professionals (general practitioners, family doctors, psychiatrists, psychologists) and two discussion groups (One with general practitioners and family doctors, and other with psychologists) as well as a national literature review on the subject. The crossing of two understandings of anxiety was identified: one belonging to the medical field, based on concepts distant from experience and another belonging to the social field, based on the experience of professionals with their patients. We conclude that their link with "sociological hypotheses" of anxiety make it possible to transform daily problems derived from the pressing socio-economic situation that patients live into problems that can be pharmacologically managed by benzodiazepines. KEYWORDS: Anxiety; Benzodiazepines; Psychopharmacology; Everyday Life. 


\section{Introducción}

Desde hace más de medio siglo los medicamentos ansiolíticos poseen un lugar destacado en el tratamiento de la ansiedad y sus síntomas. Los ansiolíticos benzodiazepínicos representan una de las principales terapéuticas farmacológicas a las que especialmente los médicos generales recurren para dar respuesta a estados de ansiedad agudos o crónicos.

El éxito de las benzodiazepinas (BDZ) es observable en las altas tasas de consumo que presentan a lo largo y ancho del globo, y en su permanencia ininterrumpida en el mercado farmacéutico desde su introducción a principios de los años sesenta. Esta permanencia se ha sostenido a pesar de las distintas críticas que han recibido como medicamentos generadores de dependencia, y da cuenta del éxito técnico y cultural de estos fármacos en la medida en que han logrado incorporarse a un tiempo a la práctica clínica y a las distintas dimensiones de la vida en sociedad (Tone, 2005).

La discusión de este fenómeno desde las ciencias sociales ha suscitado diferentes preguntas en torno a la relación existente entre la expansión del uso de las BDZ y la expansión de los estados de ansiedad. Así, es posible preguntarse si el aumento del uso de las BDZ responde a que la ansiedad presenta niveles crecientes de prevalencia a lo largo del tiempo, es decir, a un verdadero incremento de los casos de ansiedad en la población, o si su expansión responde a la estrategia de la industria farmacéutica de ampliar los límites de la categoría ansiedad, promoviendo la patologización de los distintos aspectos de la vida cotidiana (Tone, 2009). La corriente de estudios del "disease mongering", por ejemplo, ha enfatizado el modo en que los medicamentos contribuyen a la consolidación de categorías diagnósticas y su expansión en la población en general, impulsando el desarrollo de “epidemias” ficticias. Así, Moynihan, Heath y Henry (2002) entienden que los laboratorios farmacéuticos promocionan y mercantilizan las enfermedades expandiendo las fronteras de las patologías tratables por la biomedicina para abarcar a un número cada vez mayor de personas, y por lo tanto, incrementar el número de consumidores de medicamentos. Según estos autores, en este proceso, médicos, laboratorios, pacientes y medios de comunicación contribuyen a la generación de una imagen de las enfermedades como patologías graves y frecuentes que deben ser tratadas farmacológicamente.

Desde el campo de los estudios farmacéuticos, los psicofármacos son entendidos como agentes mediadores de distinciones fundamentales para el campo de la salud como lo son la propia la distinción entre la salud y la enfermedad, y la distinción entre tratamiento y mejoría (Greene \& Sismondo, 2015, p. 6), y como agentes fundamentales de los procesos de farmaceuticalización, es decir, los procesos de transformación de los problemas, capacidades y habilidades humanas en oportunidades de intervención farmacéutica (Williams, Martin, \& Gabe, 2011, p. 11).

La naturaleza inespecífica y difusa de la noción de ansiedad ha llevado a algunos a sostener la existencia de una "tradición del estrés" que engloba una serie de síntomas somáticos, psíquicos y de relacionamiento interpersonal que habitualmente surgen ante las dificultades de la vida cotidiana -tales como la fatiga, el malestar generalizado y los problemas del sueño-, que suelen explicar buena parte de la presentación de los pacientes que se reciben en la práctica de la medicina general (Horwitz, 2010). Esta misma inespecificidad ha propiciado el uso de medicamentos como el Valium(C, que no necesitan de un diagnóstico psiquiátrico definido con precisión para ser utilizados en la población en general (Frances, 2014, p. 156). De hecho, la propia industria farmacéutica, especialmente en 
los años sesenta y setenta, impulsó en los anuncios publicitarios de ansiolíticos su uso en un amplio rango de malestares, propiciando así un número importante de prescripciones de BDZ y un manejo flexible del diagnóstico de ansiedad. En Gran Bretaña, por ejemplo, a mediados de los años setenta, sólo una de ocho prescripciones de ansiolíticos se asociaba a un trastorno psiquiátrico reconocible (Abraham \& Sheppard, 1999, p. 5).

En efecto, el modo en que la profesión médica, a través de su predisposición a la prescripción de BDZ, ha convertido en blancos farmacológicos diferentes aspectos de la vida en sociedad viene siendo discutido desde al menos finales de los años setenta (Gabe, 1991). Autores fundamentales de la teoría de la medicalización, como Conrad, tomaban al Valium(C y al Librium (C como claros ejemplos del control social que la medicina ejercía sobre los comportamientos desviados (Conrad, 1979), mientras que otros señalaban que su alta prescripción no se fundamentaba en su eficacia terapéutica para el tratamiento de patologías mentales, sino en su capacidad de funcionar como herramientas paliativas de problemas sociales y personales (Waldrom, 1977). Algunos estudios en particular intentaron establecer si el uso de BZD por parte de los médicos suponía la individualización de problemas sociales colectivos y el mantenimiento del orden social estatuido (Gabe \& Lipshitz-Phillips, 1984). Más recientemente, otros investigadores sociales han enfatizado que el uso de los psicofármacos para síntomas asociados a la ansiedad no se sustenta necesariamente en el modelo de la especificidad patológica, sino en la forma en que los profesionales médicos entienden e interpretan la finalidad de los tratamientos psicofarmacológicos (Lakoff, 2004).

Este artículo, busca contribuir a la comprensión de la relación entre la expansión del uso de ansiolíticos benzodiazepínicos y el diagnóstico de la ansiedad a partir de la presentación de los resultados de una investigación realizada desde abril de 2013 a abril de 2015 . Esta tuvo como objetivo analizar el papel de la controversia sobre la utilidad clínica de las $\mathrm{BDZ}$ en el uso que se hace de ellas en las prácticas de la medicina general, la psiquiatría y la psicología en los servicios de salud pública del Uruguay. Específicamente, en este trabajo se aborda la relación entre la prescripción de BDZ y la ansiedad a partir del análisis del lazo que médicos, psiquiatras y psicólogos establecen entre ansiedad, ansiolíticos, saber psiquiátrico y técnico, y condiciones socioeconómicas de la población.

\section{Ansiedad y benzodiazepinas en el Uruguay}

Las BDZ constituyen uno de los grupos medicamentosos más utilizados a nivel mundial, ya sea como ansiolíticos o hipnóticos, y a pesar de las diferentes advertencias realizadas por el cuerpo médico y los sistemas regulatorios, continúan siendo medicamentos ampliamente utilizados en la práctica clínica (Donoghue \& Lader, 2010). A diferencia de otros países, donde es posible establecer para las BDZ importantes niveles de consumo, en el Uruguay, la ausencia de publicación de datos oficiales sobre la prescripción, venta y consumo de medicamentos en general, hace difícil poder establecer con certeza el estado de situación del uso y consumo de estos medicamentos. A pesar de ello, existen algunos indicios de que el uso de las BDZ en Uruguay comparte varias de las características que se han observado a nivel internacional.

En los años noventa, Bustelo (1994, pp. 96-99) observaba en la ciudad de Montevideo un elevado consumo de tranquilizantes menores, fenómeno significativo sobre todo en las mujeres y en la franja etaria por encima de los cuarenta años. 
Encontraba además que sólo el 31.5\% de los consumidores de tranquilizantes era portador de un trastor no psiquiátrico menor (pp. 120-123). La tasa de prevalencia mensual del 2 1,2\% era la más alta en comparación con los datos de otros países de América y Europa analizados por este autor (pp. 141-144).

Veinte años más tarde, un estudio realizado por el Observatorio de Medicamentos del Departamento de Farmacología y Terapéutica de la Universidad de la República, se propondría generar datos sobre el consumo de BDZ en todo el Uruguay para 2010, 2011 y 2012, a través de un relevamiento y análisis de datos de dispensación en farmacias de un conjunto de instituciones de salud pública y privada. Se encontró que en promedio en el período estudiado, 74 personas de cada 1000 habitantes habían consumido al menos una dosis de BDZ por día. Estos datos posicionan a Uruguay entre los países con consumos elevados. Los autores concluían que el consumo de BDZ constituye un problema de salud pública que necesita de la implementación de medidas a nivel nacional para favorecer un uso racional (Speranza et al., 2015, p. 116).

Las consecutivas encuestas nacionales de prevalencia de consumo de drogas realizadas a partir del año 2000 (Junta Nacional de Drogas, 2003, 2007, 2012, 2016), ubican los tranquilizantes como los psicofármacos con mayor prevalencia, ocupando siempre el tercer lugar en los consumos de sustancias psicoactivas, luego del alcohol y el tabaco. La última de estas encuestas muestra el valor más alto hasta la fecha: el $27.2 \%$ de la población estudiada ha consumido alguna vez en su vida tranquilizantes y su uso actual (prevalencia de consumo en los últimos 12 meses) involucra al 12,1 \% de la población total (Junta Nacional de Drogas, 2016).

La encuesta de 2015 agregaba además que del total de la población que manifestaba haber consumido BDZ, el 81,4 \% lo había hecho por prescripción médica, siendo los médicos generales quienes realizaron la prescripción en el 51,3\% de los casos contra un $37,3 \%$ de prescripciones por parte de los médicos psiquiatras. Indicaba también un mayor consumo por parte de las mujeres (Junta Nacional de Drogas, 2016).

La escasez de datos epidemiológicos sobre patologías psiquiátricas en el Uruguay no permite establecer los niveles de prevalencia de la ansiedad en la población, pero especialmente desde finales de los años setenta la noción de que los casos de ansiedad son frecuentes en la consulta médica es señalada por varios psiquiatras (Gaspar, 1978; Herrera, 1978; Murguía, 1987). A la sazón, el lazo entre ansiedad y el tratamiento ansiolítico con BDZ se había establecido en el país a través de la noción de la existencia de una psicofarmacología de la ansiedad (Herrera, 1978). Para los años 2000, siguiendo las recomendaciones del Grupo Internacional de Consenso sobre Depresión y Ansiedad (Ballenger et al., 2001), la psiquiatría uruguaya sugería suplantar las BDZ por los antidepresivos ISRS como tratamiento de primera elección en los trastornos de ansiedad (Boussard, Gaspar, Labraga, \& D'Ottone, 2002), pero las BDZ continuaron siendo uno de los principales recursos a la hora de su tratamiento (Speranza et al., 2015). 


\section{Metodología}

En esta investigación se trabajó con una metodología cualitativa pues es la adecuada para indagar valoraciones y prácticas en torno a la ansiedad y el uso de BDZ. Se recurrió al relevamiento documental, entrevistas en profundidad y grupos de discusión. Paralelamente, se relevaron artículos académicos nacionales sobre BDZ y ansiedad a partir de 1960 hasta el año 2012 en portales especializados y en bibliotecas universitarias y de sociedades científicas. Se tomó como punto de partida el año 1960 ya que antes no se encuentran publicaciones científicas sobre las BDZ, que comenzaron a llegar a Uruguay en esos años. Las fuentes relevadas fueron las siguientes: Revista de Psiquiatría del Uruguay, Revista Médica del Uruguay, Archivos de Medicina Interna, Diario Médico, Tendencias en Medicina y Cursos de Perfeccionamiento de Psiquiatría. El trabajo de recopilación se realizó desde mayo a noviembre de 2012 y se obtuvieron 86 artículos científicos, a partir de las siguientes palabras clave: ansiedad, ataques de pánico, ansiolíticos, benzodiazepinas. Esta búsqueda a través de palabras clave pudo realizarse fundamentalmente en la bibliografía publicada luego del año 2000 que es la que se encuentra digitalizada. Para los años anteriores se realizó un trabajo de archivo en las bibliotecas universitarias que disponen de las colecciones completas de las revistas científicas relevadas.

Asimismo, se entrevistaron 10 informantes calificados - seleccionados por su conocimiento del sistema de salud pública en temas de salud mental y por sus trabajos académicos en materia de prescripción y consumo de BDZ - que aportaron datos relevantes sobre la prescripción de las BDZ en Uruguay y las características específicas del sistema de salud público. Durante el año 2013, se realizaron 35 entrevistas a profesionales de la salud del sector público contratados por la Administración de Servicios de Salud del Estado (ASSE) insertos en el primer y segundo nivel de atención, de las cuales ocho entrevistas fueron a médicos generales, diez a médicos de familia, once a psiquiatras y seis a psicólogos. La pauta de entrevistas cubría cuatro tópicos: ansiedad en la clínica, prescripción de BDZ, relación con otros tratamientos farmacológicos y no farmacológicos y percepción acerca de las BDZ. Las entrevistas fueron desarrolladas en su totalidad en los lugares de trabajo de los entrevistados, tuvieron una duración promedio de 45 minutos y fueron grabadas en 33 casos y registradas de forma escrita a pedido de los entrevistados en dos casos.

En el año 2014 se realizó un grupo de discusión con seis médicos generales y médicos de familia y un grupo con cuatro psicólogas, ambos de una hora y media aproximadamente de duración. Se convocó a un tercer grupo de discusión con psiquiatras que no llegó al número mínimo de participantes. Asistieron dos profesionales cuya discusión fue considerada para el análisis junto con las entrevistas en profundidad. Como disparadores de la discusión se utilizaron artículos de la prensa uruguaya sobre ansiedad y consumo de ansiolíticos y luego se propuso discutir sobre la ansiedad en la clínica, prescripción de BDZ y su valoración. Se recogió el consentimiento libre e informado en todos los casos.

Para el análisis de los artículos se elaboró una grilla que permitió categorizar la información de acuerdo a tópicos claves en el uso clínico de las BDZ: conceptualización de la ansiedad utilizada, información científica sobre las BDZ, recomendaciones para su uso, valoración de su utilidad clínica, valoración de las prácticas de prescripción, ventajas y desventajas de las BDZ, percepción de su uso y/o prescripción como problema de salud pública, tipo de uso asociado a la ansiedad, tipo de uso asociado a 
trastornos del sueño, cambios en las indicaciones asociadas a estos medicamentos. Estos artículos fueron ingresados al programa Atlas.ti 6.1 para triangular su análisis con el de las entrevistas y los grupos de discusión.

Para el análisis de las entrevistas y de los grupos de discusión se utilizó el programa Atlas.ti 6.1 (Scientific Software Development GmbH, Berlín) y se realizó un análisis de contenido centrado en cuatro núcleos principales: ansiedad en la práctica clínica (nociones de ansiedad manejadas, percepción de la ansiedad en la consulta), acto de prescripción, relación de tratamientos farmacológicos con tratamientos no farmacológicos y valoración de las BDZ.

\section{Resultados}

Se pudo observar en las entrevistas la emergencia de una suerte de retórica sobre la ansiedad, es decir, una forma particular de presentarla discursivamente, que converge con el tratamiento dado a la ansiedad en las publicaciones académicas nacionales y mantiene puntos de contacto con las elaboraciones de la tradición psiquiátrica sobre la ansiedad pero no se limita a ellas. Esta retórica se compone de 4 tópicos principales que configuran argumentos para el uso de BDZ: (a) la ansiedad como un problema de época; (b) la ansiedad como un fenómeno omnipresente; (c) la ansiedad como un fenómeno de raíz socioeconómica; (d) ansiedad como un obstáculo para el desempeño cotidiano de los sujetos.

La ansiedad dentro de la tradición psiquiátrica es concebida como una emoción o afecto normal que puede desarrollar manifestaciones mórbidas. Se la define como una emoción desagradable, con expresiones psíquicas y fisiológicas, que surge ante situaciones peligrosas o amenazantes. Sus expresiones mórbidas se caracterizan por un exceso en la intensidad de la ansiedad experimentada en relación a los objetos o situaciones ante las que emerge. Esto convierte la distinción entre ansiedad normal y ansiedad patológica en una distinción dependiente de criterios situacionales y en uno de los problemas fundamentales de la psicopatología de la ansiedad que, si bien ha desarrollado un lenguaje descriptivo pormenorizado de la expresión patológica de la ansiedad, no ha logrado establecer una distinción neta entre ambos tipos. Las expresiones patológicas, además, son concebidas como síntomas de otros cuadros clínicos y como un cuadro mórbido independiente que puede poseer a su vez distintas variantes. Con los aportes de Freud, la ansiedad adquirió un valor explicativo central en la medida en que se la postuló como la causa de todos los cuadros neuróticos (Berrios, 2008; Horwitz \& Wakefield, 2012). Desde esta perspectiva, la "neurosis de angustia" — cuadro descripto por Freud a finales del siglo XIX — fue el diagnóstico por excelencia en torno a la ansiedad. La traducción de ansiedad por angustia como equivalentes semánticos es controversial para los psicoanalistas en el Río de la Plata. Sostener la diferenciación de ambos términos es ineludible para conceptualizar el malestar subjetivo en la actualidad como ansiedad y delimitar la función de la angustia en los procesos terapéuticos (Wainsztein, 2003). 


\section{La Ansiedad como un Problema de Época}

Una de las nociones centrales a través de las cuales los profesionales entrevistados enlazan ansiedad y BDZ, es la noción de la ansiedad como un fenómeno de actualidad. Esta noción posee una doble condición, por un lado, trata de definir la ansiedad, y por otro, trata de explicar los orígenes de la misma. Constituye de algún modo lo que llamamos una "hipótesis sociológica" sobre el origen de la ansiedad, es decir, un supuesto que los profesionales manejan para explicar las causas de la ansiedad y sobre todo sus presuntas altas tasas de prevalencia. Los profesionales recurren a su propia experiencia en el consultorio para caracterizar la prevalencia de los casos de ansiedad que reciben en su práctica clínica. Se presenta la ansiedad como muy frecuente o como la patología más frecuente: "una de las patologías más frecuentes, del diario vivir, de este mundo hoy, digo, se vive más rápido y se genera mucho (sic) más ansiedad' (MG-n2, Médico General, 49 años, sexo masculino).

La noción de la ansiedad como un fenómeno actual, vehiculiza hipótesis sociales macro, apelando a dimensiones inespecíficas de la vida en sociedad como el estilo de vida y los tiempos actuales. Algunos afirman que es un problema de la "época histórica”, lo que hace suponer que la sociedad es la que ha llevado a que la ansiedad sea la patología de nuestro tiempo. Como señala Tone (2009) el reconocimiento de una época histórica como particularmente más ansiógena que otras constituye una retórica que justifica el uso de tranquilizantes.

Entrevistada: Es un gran problema, ¿no?, yo diría que es un problema grande de toda la sociedad, ¿̇no?, de este momento, de esta época histórica. Me parece que la ansiedad, los trastornos de ansiedad, capaz que unido a la depresión y las crisis de pánico, me parece que es un gran problema de salud de nuestros tiempos, de esta época, no? preocupante diría yo. (MG-an 1, Médica General, 57 años, sexo femenino)

Es interesante señalar que esta idea aparece ya en trabajos de la década del setenta publicados en Uruguay (Herrera, 1978) y que incluso se remonta a los años cincuenta, cuando el Catedrático de Psiquiatría del momento caracterizara la ansiedad como un signo de la época (García Austt, 1950). A su vez, la percepción de la ansiedad como frecuente coincide con la literatura nacional e internacional. Por ejemplo, a nivel nacional, una publicación de difusión de temas médicos, reproducía en el año 2001 la opinión de expertos españoles que insistían en que junto con la depresión, la ansiedad era una de las patologías más frecuentes en la consulta médica (El Diario Médico, 2001). A nivel académico, Monti (2004) reiteraba la idea de que la ansiedad es uno de los trastornos más frecuentes en la comunidad y D’Ottone (2006) señalaba la ansiedad como una de las patologías más frecuentes en la consulta psiquiátrica junto a la depresión. Ya en 1987 Arduino y de los Santos, en un trabajo sobre la consulta en la emergencia psiquiátrica en un hospital general, indicaban que luego de los intentos de suicido la consulta más frecuente era lo que ellos llamaban "síndromes de ansiedad y angustia", y "síndromes depresivos". En la década siguiente los doctores Lyford-Pike y Savi (1993), al revisar los consensos internacionales de 1989 y 1991 sobre el tratamiento de trastornos de pánico, recogían la información de que para Estados Unidos, y tal vez para el mundo entero, los trastornos de ansiedad eran la patología psiquiátrica de mayor prevalencia, idea que Herrera en 1978 ya manejaba pero sin datos estadísticos que la respaldaran. 
Esta consistencia entre las producciones académicas escritas en revistas nacionales y las percepciones vertidas por los profesionales en las entrevistas en torno a la frecuencia de la ansiedad parece funcionar como un puente fundamental en el pasaje de las nociones próximas a la experiencia que se manejan sobre la ansiedad a conceptos más técnicos. Enlaza la visión de los entrevistados con el campo académico de la medicina, a pesar incluso de la falta de datos sistemáticos sobre las características de la consulta. Permite de algún modo acercar el abanico de malestares asociados a la ansiedad al terreno de las intervenciones médicas.

\section{Ansiedad Omnipresente}

La noción de la ansiedad como un fenómeno actual se emparenta con otra concepción de la ansiedad que tiende a definirla, en los dichos de los profesionales, como un fenómeno omnipresente. Existiría una ansiedad diaria, la de todo el mundo, que todos tendríamos. La idea de que la ansiedad se superpone con los miedos y preocupaciones normales y que además se encuentra en un gran número de patologías somáticas y mentales, parecería hacer de la ansiedad una categoría no enteramente adecuada a las exigencias del diagnóstico médico, que suele considerar enfermedades a aquellas entidades diagnósticas bien definidas y aisladas (Grob \& Horwitz, 2010, p. 112). Sin embargo, la noción de la ansiedad como omnipresente, tanto en la naturaleza humana como en los distintos trastornos mentales, en el discurso de los profesionales no parece desacreditarla como entidad patológica, sino, por el contrario, reforzarla en su condición de objeto de intervención médica. Aquí emergen ideas como que la ansiedad es un síntoma a la vez que un trastorno, y que como síntoma estaría en todas las patologías psiquiátricas. La ansiedad estaría presente en todos como un afecto normal pero solamente en algunos configuraría una patología. La noción de la existencia de una ansiedad saludable y otra patológica, no aparece exclusivamente en los profesionales entrevistados, sino que atraviesa el abordaje que la psiquiatría le ha dado a la ansiedad desde que la aisló como una entidad patológica a finales del siglo XIX (Berrios, 2008; Horwitz \& Wakefield, 2012).

pero a veces, bueno, ta, hay que usarlas [las BDZ]. Es decir, la ansiedad... como trastorno es un trastorno, pero no hay que olvidarse de que es un sintoma, y que es un sintoma que está en todas las patologías psiquiátricas, o sea, la ansiedad como síntoma, como ansiedad cruza todas las patologías psiquiátricas. (PSIQ-an2, Psiquiatra, 43 años, sexo femenino)

Pero justamente, los diferentes criterios para distinguir entre la ansiedad normal y la patológica son sustanciales para definir la pertinencia de un tratamiento y también para medir la prevalencia de la ansiedad (Tone, 2009, p. 3). La noción de la ansiedad como una patología legítima y además presente como síntoma en todas las patologías psiquiátricas constituye un argumento central para un uso pertinente y no banal de las BDZ (Tone, 2009, p. xix).

Esta noción de omnipresencia de la ansiedad, coincidiendo con lo planteado en investigaciones de la región (Sedronar, 2007), funciona como un argumento para el uso de las $\mathrm{BDZ}$ por parte de los médicos y también como una explicación de porqué las personas recurren a estos medicamentos. 
A mí, me sirve y mucho. $\Upsilon$ mucho, y más ahora, con todos los pacientes que todos tienen un poquitito de ansiedad o..., no o..., yo veo un beneficio total con las benzodiazepinas. Y el poder usarlas, bárbaro. (MG-al1, Médica General, 37 años, sexo femenino)

La idea de la ansiedad como algo inherente a la condición humana, así como la noción de la ansiedad como un problema de la época, se encuentra en la producción escrita sobre la ansiedad en el Uruguay desde al menos los años setenta (Herrera, 1978). También la noción de que existe una ansiedad normal y una ansiedad patológica se ve en artículos médicos, especialmente en las producciones de los años noventa (D’Ottone, 1993) y constituye una forma de argumentar la necesidad de intervenciones farmacológicas una vez que se traspasa el límite entre una y otra.

\section{Ansiedad Socio-Económica}

Otra de las nociones que constituye a la vez una caracterización de la ansiedad y una explicación de sus orígenes, es la noción de la ansiedad como un fenómeno derivado de las condiciones socioeconómicas. Con esta categoría emerge una concepción de la ansiedad como ligada a la situación social compleja de barrios y familias. Son hipótesis sociológicas que buscan explicar los casos particulares que estos profesionales reciben en la consulta, y que también dan cuenta de la alta frecuencia de los casos de ansiedad. Las actividades cotidianas, incluso pueden llegar a ser fuente de la ansiedad cuando se vive en situaciones sociales estresantes especialmente de violencia y peligro. Esta violencia se da en varios planos: es violencia doméstica, pero también es violencia entre vecinos.

Hace poco tuve un caso, porque se está dando muchos problemas de violencia acá, a nivel del barrio, tuve un caso de una, de una señora que tuvo problemas con unos vecinos, con agresiones, y con amenazas. $Y$ bueno, y realmente estaba muy ansiosa, pobre y no, y no podía dormir, y muy angustiada y eso. (MF-g4, Médica de Familia, 52 años, sexo femenino)

Se habla de "disfunción del núcleo familiar", de "situaciones familiares estresantes", que se ven agravadas por los problemas económicos. En esta apreciación sociológica de la ansiedad algunos llegan a ser detallistas y ubican la emergencia de la misma luego de la crisis económica del 2002 que asoló al Uruguay.

Y yo creo que sí, las patologías se se, van cambiando en la medida que la sociedad va cambiando, yo no creo que sea lo mismo una histeria clínica... To digo, no es lo mismo una histeria de la época de Freud que la que puede aparecer hoy, aunque tiene características muy similares, por nombrarte un cuadro. Pero yo creo que hay cosas, y yo creo que hay patologías que se han agravado por, por la realidad, por la realidad... yo siempre digo que hay un antes y un después del dos mil dos. Mm, en la crisis, en la crisis

Entrevistadora: ¿¿n qué... en la gente en qué lo vinculás?

Entrevistada: $\Upsilon$ sí, porque hubo, hubo, el cambio de las realidades socio-económicas de muchas familias afectó extremadamente, ehh, en la, justamente, en los cuadros de ansiedad y angustia, que muchas veces van juntas, yo creo que ahi fue el mayor, el mayor rebrote. (PSIC-an 1, Psicóloga, 49 años, sexo femenino) 
En síntesis, los flagelos sociales como la violencia, la drogadicción y la pobreza generan ansiedad.

Mujeres, entre los treinta y los cincuenta, con problemas familiares. Eso es lo más, ya, familiares en, hijo en la pasta base, problemas de pareja, el novio que la dejó o el marido que se fue, o, digo, ese tipo de cosas, o problemas de... este de convivencia con los hijos que ya están más grandes y que, o no aportan o no se hacen cargo de, de situaciones, o tienen problemas ellos mismos de pareja, es gente que, hijos que se han ido y vuelven a la casa paterna con, con su pro... con su familia o su media familia, digo, problemas económicos, muchísimos, y problemas laborales. (PSIQ-an 1, Psiquiatra, 55 años, sexo femenino)

También la conciencia de una vida carenciada.

la población, acá, en esta zona, es clase media empobrecida y eso genera que la gente esté angustiada, porque no puede acceder al consumismo que tenemos y es consciente de eso porque, culturalmente, tiene un buen nivel cultural. $\Upsilon$ eso te lo digo con propiedad, porque yo trabajé en una zona roja, donde la gente, realmente, vivía horrible y con mucho menos, pero era mucho menos consciente de esa falta y, por ende, era más feliz. Entonces, no tenía tanta angustia y ansiedad. (MF-p1, Médica de Familia, 52 años, sexo femenino)

Si bien estas entrevistas vinculan el desarrollo de la ansiedad a las condiciones sociales actuales, el recurso a dichas condiciones como explicación de la ansiedad es un recurso que aparece tempranamente en el discurso médico. Así, Bucknill y Tuke en su Manual de Psicología Médica publicado en 1858, señalaban que las personas podían experimentar ansiedad y aprensión como consecuencia de los bajos ingresos (citado en Berrios, 2008, p. 579).

\section{Ansiedad y Desempeño}

Otra de las nociones que permiten la transformación de la ansiedad en un blanco farmacológico es la noción de la ansiedad como un problema que afecta el desempeño del sujeto. Vale la pena destacar que esta noción aparece cuando los profesionales responden a la pregunta de cuándo la ansiedad se convierte en motivo de consulta, y también cuándo se receta por primera vez una BDZ. Con esta noción lo que queda claro es que la ansiedad pasa al campo médico cuando al sujeto que consulta, como dice uno de los técnicos, no le permite funcionar.

porque ya no le permite llevar a cabo su vida diaria, sus tareas, su funcionamientos, sus vinculos familiares, pareja, amistades, o sea que ya no puede seguir funcionando, seguir con su vida diaria, lo limita, no puede dormir, comer o come en exceso. Trae aparejado otras enfermedades. Entonces, ta, ahi ya se convierte en patológica. (MF-g2, Médica de Familia, 34 años, sexo femenino)

Este funcionamiento incluye todos los aspectos de la vida cotidiana que son resaltados en las "hipótesis sociológicas", pero principalmente en el ámbito de la familia y el trabajo. Se define así cuándo la ansiedad se convierte en patológica. Se destaca en el discurso de los profesionales la dificultad para dormir. Esta dificultad sustenta la noción de la ansiedad como una dificultad en el desempeño del sujeto, pues si no se puede dormir, 
luego no se estará en condiciones de llevar adelante un trabajo o una vida de familia satisfactoria. Esta noción, que se emparenta con la definición de "trastorno" introducida en el DSM-III y sostenida en sus ediciones posteriores, permea no sólo las narrativas de los psiquiatras, sino también la de médicos generales, médicos de familia y psicólogos. La referencia a la pérdida de capacidad de funcionamiento en áreas significativas de los sujetos es no sólo uno de los principales criterios propuestos por los distintas versiones del DSM desde 1980 para reconocer los trastornos mentales en general, sino también para reconocer los trastornos de ansiedad que aparecen incluidos como una categoría diagnóstica específica a partir del DSM-III. Estas narrativas, enlazan, por lo tanto, el tratamiento que el saber psicopatológico contemporáneo ha dado a la ansiedad con visiones acerca del funcionamiento del sujeto en sociedad. Lo que se ve afectado principalmente es entonces la esfera del "hacer", del desempeño, y algunos incluso sugieren que la llegada del sujeto al ámbito médico o psicológico se da cuando el sujeto ya no está controlando su propia vida. El sujeto sano sería entonces aquel que es capaz de acción y de control de sí mismo, en definitiva, el que no ha perdido la razón.

"Entrevistadora. ¿Cuáles serían las ventajas para el paciente de que se le prescriban benzodiazepinas?

Entrevistada: La calma de la ansiedad, la angustia. Porque una persona puede estar, yo qué sé, una, dos, tres noches sin descansar, pero después, no. Este es un mundo que la gente tiene que funcionar. (PSIQ-p2, Psiquiatra, 59 años, sexo femenino)

Esta noción de que la ansiedad llega al terreno médico cuando limita al sujeto en su accionar cotidiano también aparece en la literatura nacional. Por ejemplo, en varias publicaciones del Diario Médico (Maltzman, 2011; El Diario Médico, 2001) y en el artículo de D’Ottone en la revista Tendencias, en el que además se sostiene que la ansiedad patológica puede llevar a un funcionamiento no sólo inadecuado sino también peligroso (1993, p. 3). Podemos incluso ir más atrás en el tiempo y encontraremos esta idea, en el trabajo de Herrera de 1978 de la Revista de Psiquiatría del Uruguay.

\section{Discusión}

Esta investigación indagó las nociones que los profesionales de salud pública en Uruguay sustentan sobre la ansiedad, la vida cotidiana y su relación al uso de los ansiolíticos benzodiazepínicos. Entre estas nociones de ansiedad, fue posible distinguir algunas claramente vinculadas al saber experto y médico, que hacían referencia a las definiciones psiquiátricas de la ansiedad y aquellas que la asociaban a las condiciones actuales de vida. Ambos tipos de nociones prefiguran argumentaciones y justificaciones para el uso de los medicamentos ansiolíticos. Por un lado, la noción de la ansiedad como un fenómeno de época y la noción de la ansiedad como un fenómeno omnipresente en la vida de todo ser humano y en toda patología mental, sustentan la percepción de la ansiedad como un evento inevitable, que inunda las prácticas médica, psiquiátrica y psicológica, y naturalmente perteneciente al campo de la intervención sanitaria. Por otro lado, la noción de la ansiedad como consecuencia de las condiciones socioeconómicas y la ansiedad mórbida cuando 
afecta el desempeño del sujeto, abonan el terreno para introducir dentro de los flexibles límites del diagnóstico psicopatológico de la ansiedad los problemas y dificultades del día a día y de la vida en sociedad.

En síntesis, estas nociones de ansiedad dan cuenta de dos concepciones sobre la misma, una que la concibe claramente como perteneciente al campo médico y que para hablar de ella utiliza conceptos que Geertz (1994) denomina conceptos distantes de la experiencia, y otra que concibe la ansiedad como perteneciente al campo social y que se ampara sobre todo en la experiencia propia de los profesionales a la hora de recibir pacientes. En este plano, encontramos lo que hemos llamado "hipótesis sociológicas de la ansiedad", es decir, supuestos construidos a partir de los conceptos que Geertz llamaría cercanos a la experiencia que intentan explicar la naturaleza, el origen o la alta frecuencia de la ansiedad. Estas hipótesis obviamente no son hipótesis que provengan de un saber experto, pues este tipo de hipótesis manejadas por los profesionales son, o bien las hipótesis bioquímicas de la ansiedad o bien las hipótesis psicológicas (ya sean psicoanalíticas o conductuales). El único punto de cruce de las "hipótesis sociológicas" basadas en la experiencia y las hipótesis basadas en el saber experto podría encontrarse en la concepción de la ansiedad como un problema para el desempeño de los sujetos, pues esta idea remite sin explicitarlo a la noción de trastorno mental del DSM. En este punto de cruce que tiene lugar en la noción de ansiedad como problema de desempeño, emerge además uno de los problemas clásicos de la medicina y de la psiquiatría que es el de poder establecer una distinción entre la ansiedad normal y la patológica. En el discurso de los profesionales el umbral de lo patológico se traspasa cuando el sujeto ya no es capaz de cumplir con su funcionamiento en sociedad. Y este deber de funcionamiento social es uno de los argumentos centrales para convertir las dificultades de desempeño en blanco farmacológico y fundamentar el uso de BDZ. Este mismo lazo entre ansiedad, BDZ y funcionamiento en sociedad, fue señalado por Tone (2009) para el uso de meprobamato, otro medicamento ansiolítico, en la sociedad estadounidense de los años 50. Tone sostiene que el lazo existente entre meprobamato y estabilidad social respondía a un ethos terapéutico que promovía en primer término cambiarse a sí mismo antes que cambiar al mundo (Tone, 2009, p. 103). En este sentido, si bien las difíciles condiciones sociales de los sujetos aparecen en las "hipótesis sociológicas", no son respuestas sociales a las que van a recurrir los profesionales para erradicar la ansiedad, sino respuestas farmacológicas.

Las hipótesis sociológicas, además, son de dos órdenes: uno en el que la sociedad es considerada un proceso macro que por sus características es en sí misma una sociedad "ansiógena” y otro en el que factores sociales más específicos son interpretados por los profesionales como causa de la ansiedad (por ejemplo, la vida de familia, la situación económica apremiante). Según Pignarre (2003) las hipótesis sociológicas, que intentan explicar a través de la sociedad la expansión de los disturbios mentales, son uno de los argumentos también utilizados para explicar el aumento de los diagnósticos de depresión. La sociedad sería cada vez más "depresógena” provocando grandes niveles de sufrimiento social. Lo interesante aquí es notar, como señala Lucas (2004), que no todas las patologías se convierten en el vehículo para representar una "enfermedad social” y sí lo hace la ansiedad. La ansiedad entonces puede ser concebida como la enfermedad de época, la enfermedad de la sociedad en crisis uruguaya, la enfermedad que representa el malestar del estilo de vida del país. Es nuevamente la flexibilidad de la categoría diagnóstica de ansiedad, que cubre un amplio gradiente de síntomas y malestares, la que hace posible esta vehiculizaci- 
ón en la ansiedad de la idea de una sociedad mórbida. Asimismo, esta noción de ansiedad indefectiblemente enlazada a interpretaciones sociológicas de la misma, dan cuenta de un uso de las BDZ amparado por cierta flexibilidad interpretativa, no sólo sobre aquello que puede ser englobado bajo la noción de ansiedad, sino también acerca de las funciones o fines técnicos de estos medicamentos. En rigor, las $\mathrm{BDZ}$ aparecen definidas no sólo como medicamentos para solucionar problemas de salud mental, sino también para solucionar problemas sociales.

En este sentido, la flexibilidad interpretativa en el uso de las BDZ parecería encontrar puntos de contacto con los usos de psicofármacos que en otros países de la región se han descrito en contex to de crisis socioeconómicas. Por ejemplo, como sucedía en la Argentina de la crisis financiera del año 2001 con los medicamentos antidepresivos (Lakoff, 2004), los usos de las $\mathrm{BDZ}$ parecen no estar respaldados por una concepción biológica de los trastornos mentales ni por un diagnóstico preciso del trastorno de ansiedad. Por el contrario, las interpretaciones que hemos llamado "sociológicas" presentadas por los profesionales acerca de la ansiedad, respaldan un uso de las BDZ para permitir el funcionamiento vital en situaciones de vulnerabilidad social.

De esta manera, la clínica de la ansiedad y el uso de las BDZ que se hace en ella posibilitan la redefinición de los problemas de la vida cotidiana en términos de la dolencia ansiedad y, por consiguiente, su transformación en blancos farmacológicos. Como señalan Dumit y Greenslit (2006), asistimos al afianzamiento de una "cultura farmacéutica" que impulsa la amalgama de las experiencias personales y subjetivas con la noción de una vida en sociedad inseparable del consumo de medicamentos.

\section{Referencias}

Abraham, J. \& Sheppard, J. (1999). The therapeutic nightmare: the battle over the world's most controversial sleeping pill. Londres: Earthscan.

Arduino, M. \& de los Santos, O. (1987). La emergencia psiquiátrica en el Hospital General. Revista de Psiquiatría del Uruguay, 52(308), 67-77.

Ballenger, J. C., Davidson, J. R. T., Lecrubier, Y., Nutt, D.J., Borkovec, T. D., Rickels, K., Stein, D. J., Wittchen, H. U. (2001). Consensus Statement on Generalized Anxiety Disorder From the International Consensus Group on Depression and Anxiety. The Journal of Clinical Psychiatry, 62(11), 53-58.

Berrios, Gr. (2008). Historia de los síntomas de los trastornos mentales. La psicopatología descriptiva del siglo XIX. México: Fondo de Cultura Económica.

Boussard, M., Gaspar, E., Labraga, P., \& D'Ottone, A. (2002). Pautas terapéuticas de trastornos de ansiedad. Revista de Psiquiatría del Uruguay, 66(22), 130-152.

Bustelo, P. (1994). Estudio de la prevalencia de tranquilizantes menores en la ciudad de Montevideo. Tesis de doctorado en Psiquiatría, Escola Paulista de Medicina. San Pablo, Brazil.

Conrad, P. (1979). Types of medical social control. Sociology of Health and Illness, 1(1), 1-11. Donoghue, J. \& Lader, M. (2010). Usage of benzodiazepines: A review. International Journal of Psychiatry in Clinical Practice, 14(2),78-87.

D'Ottone, A. (1993). Ansiedad: diagnóstico y conducta en medicina gral. Tendencias, 2, 3-14. 
D’Ottone, A. (2006). La psiquiatría actual en el contexto médico. Tendencias en Medicina, 15(9), 103-110.

Dumit, J. \& Greenslit, N. (2006). Informated Health and Ethical Identity Management. Culture, Medicine and Psychiatry, 30(2), 127-134.

El Diario Médico. (2001, octubre). Atención primaria y depresión. El Diario médico, 7.

Frances, A. (2014). Saving Normal. An Insider's Revolt Against Out-of-Control Psychiatric diagnosis, DSM-5, Big Pharma, and the Medicalization of Ordinary Life. New York: William Morrow.

Gabe, J. (1991). Personal troubles and public issues: the sociology of long-term tranquilliser use. In Understanding tranquillisers use. The roles of social sciences. Sociology Review, 34(4), 737-772.

Gabe, J. \& Lipshitz-Phillips, S. (1984). Tranquilisers as social control? Sociological Review 32(3), 524-546.

García Austt, E. (1950). Neurosis y vida moderna. Revista Uruguaya de Psiquiatría, 90, 3-8. Gaspar, E. (1978). Ansiedad y angustia en la clínica psiquiátrica. In Facultad de Medicina, Escuela de Graduados. Primer curso de perfeccionamiento para psiquiatras del cono sur. Actualización terapéutica psicofarmacológica (pp. 43-52). Montevideo: Universidad de la República. Geertz, C. (1994). Desde el punto de vista del nativo: sobre la naturaleza del conocimiento antropológico. In Conocimiento local. Ensayos sobre la interpretación de las culturas (pp. 7390). Barcelona: Paidós.

Greene, J. A. \& Sismondo, S. (2015). Introduction. In The Pharmaceutical Studies Reader (pp. 1-16). Chichester: Wiley-Blackwell.

Grob, G. \& Horwitz, A. (2010). Transforming Amorphous Stress into Discrete Disorders: The Case of Anxiety. In Diagnosis, therapy, and evidence: conundrums in modern American medicine (pp. 111-140). New Jersey: Rutgers University Press.

Herrera, J. (1978). Psicofarmacología de los estados de ansiedad, Revista de Psiquiatría del Uruguay, 43(258), 215-232.

Horwitz, A. V. (2010). How an age of anxiety became an age of depression. Milbank Quarterly, $88(1), 112-138$.

Horwitz, A. \& Wakefield, J. (2012). All we have to fear. Psychiatry's transformation of natural anxieties into mental disorders. New York: Oxford University Press.

Junta Nacional de Drogas. (2003). In III Encuesta Nacional de Hogares sobre Consumo de Drogas. Recuperado de https://www.gub.uy/junta-nacional-drogas/

Junta Nacional de Drogas. (2007). In 4a Encuesta Nacional en Hogares sobre consumo de drogas. Recuperado de https://www.gub.uy/junta-nacional-drogas/comunicacion/publicaciones/iv-encuesta-nacional-en-hogares-sobre-consumo-de-drogas-2006

Junta Nacional de Drogas. (2012). In 5 a Encuesta Nacional en Hogares sobre consumo de drogas. Informe de Investigación. Recuperado de https://www.gub.uy/junta-nacional-drogas/ comunicacion/publicaciones/v-encuesta-nacional-en-hogares-sobre-consumo-de-drogas-2011 Junta Nacional de Drogas. (2016). In VI Encuesta Nacional de Hogares sobre Consumo de Drogas. Recuperado de https://www.gub.uy/junta-nacional-drogas/comunicacion/publicaciones/vi-encuesta-nacional-en-hogares-sobre-consumo-de-drogas-2016

Moynihan, R., Heath, I., \& Henry, D. (2002). Selling sickness: the pharmaceutical industry and disease mongering. British Medical Journal, 324(7342), 886-890.

Lakoff, A. (2004). The Anxieties of Globalization: Antidepressant Sales and Economic Crisis in Argentina. Social Studies of Science, 34(2), 247-269.

Lucas, R. (2004). In and Out Culture: Ethnographic Means to Interpreting Schizophrenia. In J. H. Jenkins \& R. J. Barrett (Eds.), Schizophrenia Culture, and Subjectivity. The Edge of Experience (pp. 184-190). Cambridge: Cambridge University Press. 
Lyford-Pike, A. \& Savi, G. (1993). Actualización del tratamiento del trastorno por pánico, Revista de Psiquiatría del Uruguay, 57(326), 31-35.

Maltzman, D. (2011, mayo). Trastorno de pánico. El Diario médico, 14(130), 4. Recuyperado de http://eldiariomedico.com.uy/diarios/a14/diario\%20130.pdf

Monti, J. (2004). Alteraciones del sueño en los trastornos de ansiedad generalizada y su tratamiento, Revista de Psiquiatría del Uruguay, 64(2), 286-300.

Murguía, D. (1987). Psicofarmacología de la ansiedad, Revista de Psiquiatría del Uruguay, 52(309-310), 206-212.

Speranza, N., Tamosiumas, G., Toledo, M., Olmos, I., Artagaveytia, P., Domínguez, V., \&Pagano, E. (2015). "Consumo de benzodiazepinas en la población uruguaya: un posible problema de salud pública”. Revista médica uruguaya. 31(2), 112-119.

Pignarre, P. (2003). La depresión: una epidemia de nuestro tiempo. Barcelona: Debate.

Sedronar. (2007). El consumo indebido de medicamentos psicotrópicos en la vida cotidiana. Un estudio exploratorio sobre representaciones sociales y patrones de uso. Buenos Aires. Recuperado de http://www.observatorio.gov.ar

Tone, A. (2005). "Listening to the past: History, psychiatry, and anxiety". Canadian Journal Psychiatry, 50(7), 373-380.

Tone, A. (2009). The Age of Anxiety: A History of America's Turbulent Affair with Tranquilizers. New York: Basic Books.

Wainsztein, S. (2003). La ansiedad: su manifestación clínica. Espacio de trabajo de la Escuela Freudiana de Buenos Aires. Foro de Psicoanálisis del 29/04/2003. Recuperado de http://www. efba.org/efbaonline/wainsztein-13.htm

Waldrom, I. (1977). Increased prescribing of Valium, Librium, and other drugs--an example of the influence of economic and social factors on the practice of medicine. International Journal of Health Services, 7(1), 37-62.

Williams, S., Martin, P, \& Gabe, J. (2011). The pharmaceuticalisation of society? A framework for analysis. Sociology of Health \& Illness, 33(5), 710-725.

\section{ANDREA BIELLI \\ http://orcid.org/0000-0002-7732-7584}

Profesora Agregada del Instituto de Psicología Clínica, Universidad de la República, Montevideo, Uruguay. Doctora por el programa de Filosofía, Ciencia, Tecnología, Sociedad del País Vasco.

E-mail: abielli@psico.edu.uy

\section{PILAR BACCI}

http://orcid.org/0000-0002-6611-1905

Profesora Adjunta del Instituto de Psicología Clínica. Magíster en Psicología Clínica por la Universidad de la República, Montevideo, Uruguay.

E-mail: pilarb@psico.edu.uy 


\section{GABRIELA BRUNO \\ http://orcid.org/0000-0002-7428-0442}

Profesora Adjunta del Instituto de Psicología Clínica. Magíster en Psicología Clínica por la Universidad de la República, Montevideo, Uruguay.

E-mail: gbrunocamares@psico.edu.uy

\section{NANCY CALISTO \\ http://orcid.org/0000-0002-6317-1531}

Asistente del Instituto de Psicología Clínica. Magíster en Psicología Clínica de la Universidad de la República, Montevideo, Uruguay.

E-mail: ncalisto@psico.edu.uy

\section{SANTIAGO NAVARRO}

http://orcid.org/0000-0002-7642-303X

Licenciado en Psicología. Magíster en Psicología Clínica de la Universidad de la República, Montevideo, Uruguay.

Ayudante del Instituto de Psicología Clínica.

E-mail: santiago.navarro.psi@gmail.com

$\begin{array}{ll}\text { Histórico } & \begin{array}{l}\text { Submissão em: 11/03/2017 } \\ \text { Revisão em: 04/08/2017 } \\ \text { Aceite em: } 27 / 06 / 2019\end{array} \\ & \text { Concepción : AB } \\ & \text { Recolección de datos : AB; PB; GB; NC; SN } \\ & \text { Análisis de datos : AB; PB; GB; NC; SN } \\ & \text { Redacción del manuscrito : AB; PB; GB; NC; SN } \\ & \text { Crítica - revisiones de contenido intelectual importante : } \\ \text { AB; PB; GB } & \text { Aprobación final del manuscrito : AB } \\ \text { Contribuición de los autores } & \text { La investigación en la que se basa este artículo recibió } \\ & \text { apoyo financiero del Programa de Proyectos de I+D de } \\ \text { la Comisión Sectorial de Investigación Científica (CSIC) } & \text { de la Universidad de la República, llamado 2012. } \\ \text { Agencia de financiación } & \text { No aplicable } \\ \text { Consentimiento de uso } & \text { La investigación en la que se basa este artículo contó con } \\ \text { de imagen } & \text { la aprobación del Comité de Etica en Investigación de la } \\ & \text { Facultad de Psicología, Universidad de la República. Se } \\ \text { recogió el consentimiento libre e informado en todos los } \\ \text { casos. }\end{array}$

